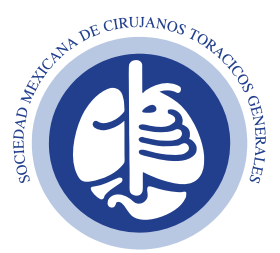

Vol. 1, Núm. 3

Septiembre-Diciembre 2020 pp 85-90

\title{
El cirujano de tórax como líder en la reconversión de un área hospitalaria para la atención de pacientes con SARS-CoV-2
}

The thoracic surgeon as a leader in the reconvertion of
a hospital area for the care of patients with SARS-CoV-2

Miguel Martínez-Arias, ${ }^{*}$ Fuensanta Domínguez-Garduño, ${ }^{\ddagger}$ Maribel Arzate-Plata ${ }^{\ddagger}$

Palabras clave:

Cirujano de tórax,

SARS-CoV-2, COVID-19,

administración hospitalaria.

Keywords:

Thoracic surgeon,

SARS-CoV-2, COVID-19,

hospital administration.

* Jefatura de Cirugía de

Tórax y Neumología.

₹ Unidad de Desarrollo

y Calidad.

Servicio de Cirugía de Toráx. Centro Médico ISSEMyM.

Recibido: 22/08/2020

Aceptado: 25/08/2020

Correspondencia:

Dr. Miguel Martínez-Arias

E-mail: drmartzari@

hotmail.com

\begin{abstract}
RESUMEN
México atraviesa en estos momentos la epidemia de mayor impacto de todos los tiempos para nuestro sistema de salud. La reconversión hospitalaria representa un reto del personal hospitalario y administrativo, que el neumólogo y el cirujano de tórax deben afrontar. En este documento se muestra una breve reseña de nuestra experiencia en la reconversión de un área hospitalaria para la atención de pacientes con SARS-CoV-2 en población derechohabiente.
\end{abstract}

\section{ABSTRACT}

Mexico is currently going through the pandemic with the greatest impact of all times in the current health system. Hospital reconversion represents a challenge for hospital and administrative personnel, the pulmonologist and the thoracic surgeon must face it. This document shows a brief review of our experience in the reconversion of a hospital area for the care of patients with SARS-CoV-2 in the eligible population.

\section{INTRODUCCIÓN}

El 31 de diciembre de 2019, las autoridades de Wuhan en la provincia de Hubei en China, con población de 12 millones de personas y la capital de Hubei, al sureste del país, con población de 56 millones de personas, reportaron un conglomerado de 27 casos de síndrome respiratorio agudo de etiología desconocida entre personas vinculadas a un mercado húmedo (de productos marinos). Siete pacientes fueron reportados como casos severos. ${ }^{1}$ El 9 de enero se anunció de manera oficial que la causa era un virus que inicialmente fue llamado nuevo Coronavirus-2019. El 11 de febrero, posterior al análisis genómico, se nombró oficialmente a la enfermedad causada como COVID-19 (coronavirus disease); el virus se nombró como SARS-CoV-2 por su homología genética. El 11 de marzo la Organización Mundial de la Salud (OMS) declaró la pandemia por SARS-CoV-2. ${ }^{2}$

Citar como: Martínez-Arias M, Domínguez-Garduño F, Arzate-Plata M. El cirujano de tórax como líder en la reconversión de un área hospitalaria para la atención de pacientes con SARS-CoV-2. Rev Mex Cir Torac Gen. 2020; 1(3): 85-90. https://dx.doi. org/10.35366/101476 


\section{Medidas tomadas en China}

Las medidas tomadas por China fueron catalogadas como brutales, pero efectivas: fue cerrado completamente el transporte público, tanto al interior de Wuhan como en el resto de las ciudades, sin excepción para ninguna persona, inclusive para emergencias médicas, escuelas y universidades. A esto se sumaron centros comerciales y tiendas, con excepción de comercios de comidas y medicamentos, sólo se podía circular en la calle con permisos especiales, se limitaban a permitir la salida a un familiar cada dos días para comprar necesidades básicas. La última fase de estas medidas se presentó cuando el servicio de policía tenía la obligación de visitar casa por casa realizando una inspección del estado de salud, forzando al aislamiento individualizado en caso de síntomas, se monitorizaron las calles mediante drones y se decretó el uso obligatorio de cubrebocas para toda la población..$^{3-5}$

Las medidas aplicadas en China no fueron suficientes para evitar que el virus se propagara en todo el mundo, en la actualidad se reportan 82,992 casos diagnosticados con 4,634 muertes registradas ${ }^{6}$ y con un índice de letalidad de $5.5 \%$. Siendo el país en donde se detonó la pandemia actual estos datos son significativos, ya que no contaban con experiencia en el manejo y la aplicación de las medidas ante este nuevo virus SARS-CoV-2.

\section{Medidas tomadas en México}

México reportó el primer caso confirmado el 28 de febrero de 2020,7 éste se presentó en la Ciudad de México posterior a un viaje del paciente a Italia (país que reportaba hasta ese momento el mayor número de casos confirmados en el mundo), en consecuencia los siguientes tres casos reportados provenían de este mismo país, motivo por el cual la Secretaría de Salud emitió el documento por el cual se establece la definición operacional de caso sospechoso con criterios clínicos en los últimos 14 días y que además en el mismo período de tiempo refiriera haber viajado a sitios de transmisión activa. ${ }^{8}$

La Secretaría de Salud de México emite el 23 de marzo el documento en el que recomienda suspender temporalmente las actividades no esenciales de los sectores público, social y privado, autorizando el resto de las actividades que incluyen el transporte público, eventos de concentración menores a 5,000 personas, así como el libre tránsito en la vía pública. ${ }^{9}$ El 29 de marzo la Secretaría de Salud, a través de la Comisión Coordinadora de Institutos Nacionales de Salud y Hospitales de Alta Especialidad, presentan el programa de reconversión hospitalaria COVID-19, el cual tiene por objetivo coordinar e integrar la respuesta hospitalaria para la atención de pacientes graves por COVID-19. Éste incluye tres fases basadas en la ocupación hospitalaria: en la primera fase se incluyeron a 13 hospitales, en su mayoría institutos, ${ }^{10}$ para después incorporar al resto de hospitales de cada institución de servicios médicos, teniendo como prioridad a los hospitales que tengan mayor capacidad de atención.

La misma Secretaría de Salud reportó el número de unidades médicas del Sistema Nacional de Salud en operación con un total de 121,435 camas para hospitalización y 230 unidades de cuidados intensivos con un total de 1,553 camas disponibles, además de 145 áreas de cuidados intermedios con un total de 952 camas disponibles, 2,053 ventiladores y 5,335 monitores. En cuanto a recursos humanos se reportaron un total de 37,596 médicos generales, 112,042 enfermeras de contacto, 1,284 urgenciólogos, 207 neumólogos y 174 infectólogos; para el valle de Toluca se reportaron un total de 62 unidades de terapia intensiva, de las cuales el Centro Médico ISSEMyM cuenta con 12 de ellas, 145 ventiladores, de los cuales 22 eran parte del Centro Médico ISSEMyM y con una capacidad en ese momento de 55 camas, de las cuales 44 pertenecían al cuarto piso de Medicina Interna y las 11 restantes a Terapia Intensiva. ${ }^{11}$

\section{MATERIAL Y MÉTODOS}

\section{Centro Médico ISSEMyM Toluca}

Ante el inminente escenario se iniciaron juntas de trabajo a partir de enero de 2020 con el objetivo de estar preparados. El sistema de salud de dicha institución ofrece servicios de salud y prestaciones sociales a 1,140,072 derechohabientes, cuenta con una plantilla de 7,379 servidores públicos dentro de las 112 unidades médicas, ${ }^{12}$ siendo este hospital el de mayor capacidad debido a que puede brindar atención que involucra altas especialidades médicas.

Las juntas de trabajo realizadas previamente a la reconversión del segundo piso del Centro Médico 
ISSEMyM Toluca sirvieron para establecer una forma de trabajo con el objetivo de brindar al paciente la atención necesaria y a su vez otorgar al personal de salud la completa seguridad para el desempeño de sus funciones. Esta labor incluía también establecer una logística de trabajo funcional, práctica y que cumpliera con las recomendaciones establecidas por la OMS/OPS y buenas prácticas establecidas en diferentes centros hospitalarios que ya contaban con experiencia. Sin embargo, los datos reportados a nivel mundial sobre los contagios del personal hospitalario representaban un reto, ya que tendríamos que mejorar situaciones que pudieran poner en riesgo al personal hospitalario.

\section{SARS-CoV-2.}

La OMS estima que la tasa de contagio (R0) del virus SARS-CoV-2 es de 1.4 a $2.5,,^{13}$ aunque otras estimaciones hablan de un rango entre 2-3. ${ }^{14}$ Un valor de R0 inferior a 1 indica una escasa capacidad de extensión de una enfermedad infecciosa, mientras que valores de R0 superiores a 1 indican la necesidad de emplear medidas de control para limitar su extensión. La vía de transmisión del COVID-19 es por contacto y gotas respiratorias (aerosoles/gotículas) en distancias cortas (1.5 m) y también por fómites contaminados por dichos aerosoles, no se puede descartar completamente que exista cierto grado de transición por vía área.

El contacto prolongado es el mayor riesgo. ${ }^{15-17}$ La reconversión del segundo piso del Centro Médico ISSEMyM Toluca estaba conformada con el personal médico asignado a la coordinación quirúrgica, siendo las especialidades quirúrgicas propiamente dichas las que cuentan con menor experiencia en el manejo de este tipo de pacientes, por lo que se determinó que solamente los pacientes contagiados por SARS-CoV-2 moderados no ventilados se estarían atendiendo en esta área. Sin embargo, el paciente no ventilado representaba un mayor riesgo de exposición debido a la producción de aerosoles y gotículas, lo cual, como ya se mencionó, representaba un reto para conformar la estructura de esta nueva área COVID-19.

\section{Contagio del personal hospitalario}

El Hospital de Tongji, en Wuhan, reportó que posterior a realizar pruebas a 9,684 trabajadores de la salud,
110 dieron positivos, con tasa de contagio de 1.1\%, $71.8 \%$ mujeres, con rango de edad de 30-47 años, la prevalencia de infección subclínica reportada fue de $0.74 \%{ }^{18}$ Reino Unido reportó contagio de 30.5\% del personal hospitalario a nivel nacional, incluyendo trabajadores de la salud que se encontraban en la primera línea y segunda línea, con un crecimiento diario a tasa de $9.1 \%$ desde el inicio de la reconversión hospitalaria en dicho país. La Comisión Nacional de Salud de China reportó 3,300 trabajadores de la salud infectados, $4 \%$ del total reportado hasta ese momento $(81,285){ }^{19}$

España al término de marzo reportó 6,500 contagios en el personal de salud, lo que representaba $13.6 \%$ del total de casos; ${ }^{19}$ en Italia el Consejo Internacional de Enfermería (ICN) en conjunto con la Asociación Italiana de Enfermeras (CNAI) reportaron el 9\% de contagios en el personal de enfermería a nivel nacional. ${ }^{20}$ En México, la Secretaría de Salud reportó a inicios de mayo 8,544 contagios del personal de salud, de los cuales 111 fallecieron $(23.51 \%$ del total de contagios para ese momento), $41 \%$ del personal de enfermería, $37 \%$ de médicos, $19 \%$ de profesionales de la salud, $2 \%$ de laboratoristas y $1 \%$ de dentistas. ${ }^{21}$

\section{Infraestructura previa del área de reconversión}

La área destinada para la ampliación de camas para el manejo de pacientes con SARS-CoV-2 cuenta con dos alas que se encuentran unidas por una central de trabajo, esta área central cuenta con un espacio para la preparación de medicamentos, almacén de soluciones, insumos básicos y monitoreo, así como un área para el proceso de desinfección del material hospitalario. En la zona central que divide las dos alas está el área de trabajo de médicos, oficinas y dos baños externos; la capacidad total de este piso es de 48 camas.

Tabla 1: Aislamiento e infraestructura.

- Escuchar las expresiones de resistencia y mostrar empatía

- Generar información de hechos, necesidades, objetivos y efectos del cambio

- Hacer ajustes a la manera de implementar el cambio

- Reducir la incertidumbre y la inseguridad

- Buscar apoyos que aumentan la credibilidad

- No imponer el cambio

- Establecer compromiso común 


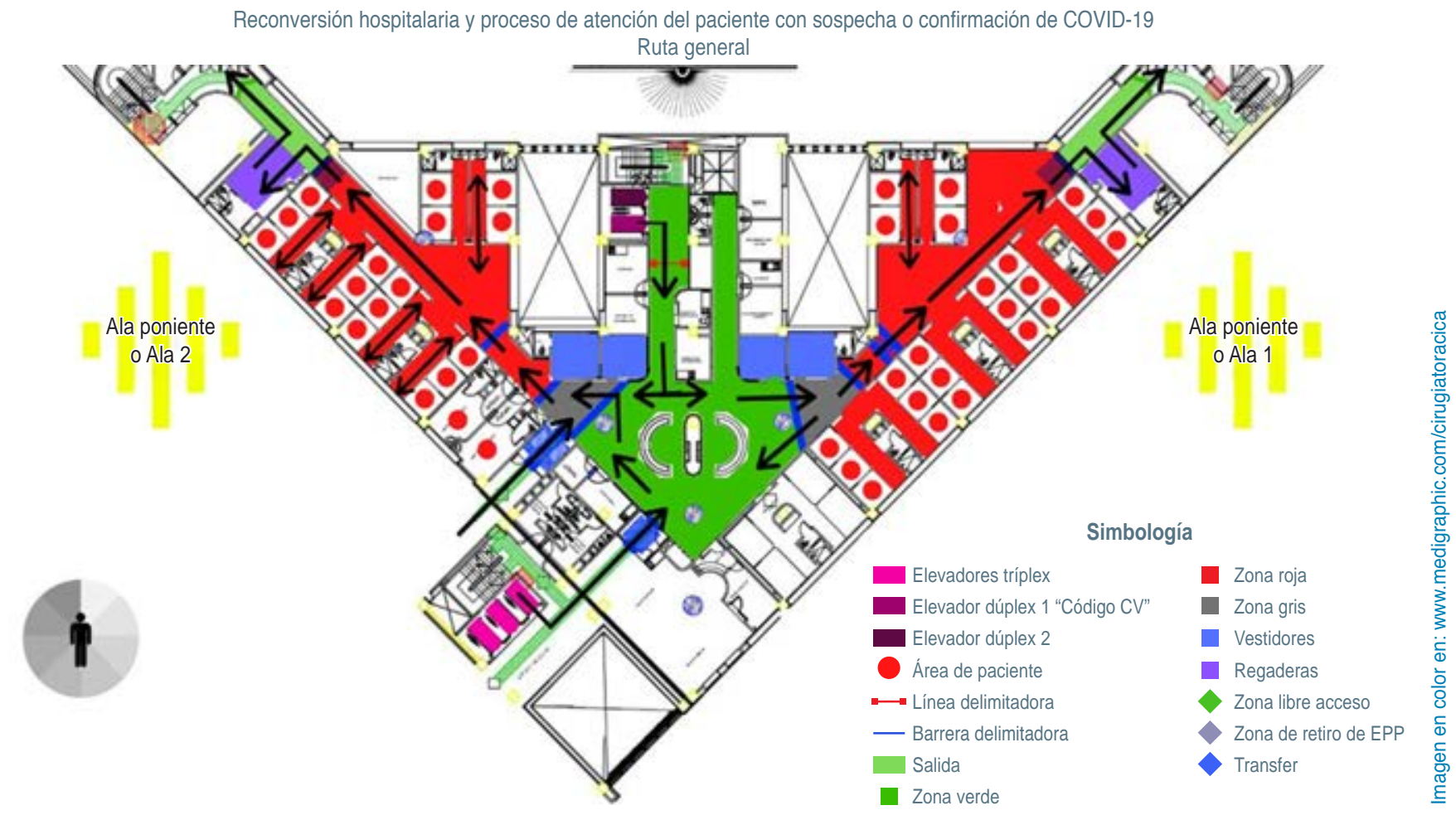

Figura 1: Esquema del área reconvertida con simbología.

\section{Planeación}

Se abordó esta situación con un enfoque de administración por objetivos, por lo que, como parte de la planeación, se establecieron estas metas a alcanzar del área COVID. ${ }^{22}$ Ésta tenía que ser funcional dando la posibilidad de que el personal hospitalario pudiera cumplir con sus actividades con la seguridad suficiente para realizarlas. La logística de trabajo establecida debía ser clara, otorgando al personal médico y enfermería actividades preestablecidas. La organización fue llevada a cabo mediante mesas de trabajo, incluyendo departamentos de enfermería, administración, trabajo social, calidad, epidemiología y arquitectura, estas mesas de trabajo fueron dirigidas por el Servicio de Cirugía de Tórax y Neumología, el cual se encuentra asignado a la coordinación quirúrgica del Centro Médico.

\section{Reconversión}

Aprovechando la estructura hospitalaria se determinó separar las dos alas aislándolas completamente una de la otra, lo cual permitió que el área central sirviera para trabajo de enfermería y médicos; fue necesario equipar el área central con máquinas de computación para trabajo y visualización de imágenes.

En cuanto a la capacidad de las 48 camas, cuatro camas se eliminaron ya que los espacios fueron utilizados para cuartos de vestido y dos para resguardo de ropa limpia, dando una capacidad total restante de 44 camas, de las cuales se destinó el 10\% para la atención del personal hospitalario contagiados por el virus, dos camas de las 40 restantes se encuentran separadas cada una, utilizando estas habitaciones para los pacientes con infecciones nosocomiales agregadas por agentes multidrogorresistentes (MDR), 38 restantes fueron concentradas en cinco cubículos de seis camas y cuatro cubículos de dos camas, las cuales, estructuralmente, compartían el espacio físico, cada cubículo comparte un lavamanos y un baño completo.

Para llevar a cabo la reconversión se determinaron diferentes medidas de aislamiento y mejoras en la infraestructura que se exponen en la Tabla 1 y Figura 1, estas medidas se encuentran alineadas con los objetivos estructurados desde el inicio. 


\section{RESULTADOS}

\section{Implementación}

Dentro del comportamiento organizacional según Chavenato I. ${ }^{23,24}$ se entienden dos tipos de decisiones: las programadas y las no programadas. Partiendo de la base en la que ninguna institución de salud del mundo se encontraba preparada, fue necesaria la toma de medidas de tipo no programadas, lo cual llevó a un arduo trabajo e implementación de medidas para disminuir la resistencia al cambio dentro de los trabajadores (Tabla 2). ${ }^{24}$

La coordinación de esta área fue manejada en conjunto con el Servicio de Cirugía General, Cirugía de Tórax y Neumología; por las medidas tomadas por la Secretaría de Salud junto con el Consejo de Salubridad, que determinó incapacitar a los grupos vulnerables del personal médico y paramédico, se dejó de contar con aproximadamente el $40 \%$ de nuestro personal. ${ }^{25}$ Por esta razón, tomando las estrategias organizacionales y las categorías que según Ansoff hablan del máximo rendimiento, responsabilidad social y actitud frente a los riesgos, ${ }^{26}$ se implementó un coordinador en el área central con equipo de médicos y enfermeras destinados para la atención y el paso de visita en las alas de hospitalización. Este coordinador tiene la función de recibir el estatus diario del paciente, realizar cambios, decidir altas, así como ingresos y traslados, los controles llevados a cabo fueron establecidos mediante el personal hospitalario, quien se autorregula al detectar malas prácticas del retiro del equipo de protección personal (EPP), fallas en el flujo del personal, así como fallas detectadas en el flujo de entrada de alimentos,

Tabla 2: Áreas de reconversión hospitalaria.

- Unidades de camas de hospitalización (alas) separadas por barreras de aislamiento (2)

- Zona verde área amortiguadora de protección

- Zona azul (2) de restricción y funcionamiento (cuartos de vestido)

- Zona gris (2) para el acceso y la circulación interna

- Zona roja (2) para la hospitalización con áreas de trabajo Enfermería y médicos equipados (equipo de cómputo, carro de paro) y área de descanso para personal de salud en área COVID

- Zona de retiro del equipo de protección personal (EPP) (2)

- Aislamiento individualizado mediante cortinillas con ventanilla y flujo de aire circulante (44)

- Áreas para aseo, baño y descanso del personal hospitalario (2) ruta de residuos peligrosos biológico-infecciosos (RPBI) y camilleros.

Para el control de contagio del personal hospitalario se está llevando a cabo un registro epidemiológico, todo el personal hospitalario es monitoreado con base en su temperatura a la entrada. Al momento, bajo la revisión de controles epidemiológicos, no se tiene registro de contagio en el personal de trabajo de esta área; sin embargo, cabe mencionar que no se están realizando pruebas sistemáticas, siendo éstas reservadas para el personal hospitalario con síntomas, esto debido a que la toma de pruebas aleatorias podría desencadenar una ausencia de personal mayor de la que ya existe.

\section{DISCUSIÓN}

México atraviesa en estos momentos la pandemia de mayor impacto dentro del sistema de salud, la problemática en salud se calcula, por parte del Instituto Tecnológico de Massachusetts (MIT), que será de proporciones tan grandes que $1.7 \%$ de la población mexicana se infectará a finales de agosto y $0.1 \%(132,446)$ de la población total del país morirá a causa del virus SARS-CoV-2. ${ }^{27}$

Aunque para ciertas regiones del mundo como España, Italia y China la curva de casos se encuentra ya rebasando el pico máximo de infectados y que el reporte en breve será que los hospitales ya no se encontraran en ocupación máxima, en el continente americano estamos enfrentando un reto de proporciones titánicas: Estados Unidos se encuentra en su peor crisis sanitaria en la historia con un total de 1,797,232 casos confirmados y 104,633 muertes reportadas; México, al término de este documento, reporta 84,627 casos confirmados, situándose en el $15^{\circ}$ lugar de mayores casos en el mundo, superando los casos de China, sitio de origen de la pandemia, (82,999 casos reportados). ${ }^{28}$

La reconversión de áreas hospitalarias para ampliar la capacidad es un reto al cual nos estamos enfrentando de manera continua debido a la magnitud de contagios activos. El cirujano de tórax y la neumología llevan un camino paralelo, no sólo durante la preparación, sino también durante el desempeño de sus funciones profesionales. En la actualidad, el perfil del cirujano de tórax debe contar con estudios de posgrado dirigidos a la administración hospitalaria, ya que un importante 
número de especialistas en la materia se encuentran bajo cargos directivos en diferentes instituciones a lo largo del territorio nacional; por lo tanto, representa una responsabilidad para nuestro gremio la preparación y la especialización constante.

\section{CONCLUSIÓN}

El conjunto de esfuerzos humanitarios y científicos bajo la coordinación y conducción de personal calificado podrá hacerle frente al reto de seguridad social más grande en la historia de nuestro país.

"Es el momento de ser prudentes, no entrar en pánico. De la ciencia, no del estigma. De los hechos, no del miedo." (Guterres A.) ${ }^{29}$

\section{REFERENCIAS}

1. Organización Panamericana de la Salud (OPS), Organización Mundial de la Salud (OMS). Alerta epidemiológica OPS/OMS: Nuevo coronavirus (nCoV). 16 de enero de 2020.

2. Guerrero J, Victal G, Martínez M. Guía práctica COVID-19 EDOMEX. 2020.

3. Li X, Zaho X, Sun Y. The lockdown of Hubei province causing different transmission dynamics of novel coronaries (2019-nCov) in Wuhan and Beijing. medRxiv. 2020.

4. Yuan Z, Xiao Y, Dai Z, Huang J, Chen Y. A simple model to assess Wuhan lock-down effect and region efforts during COVID-19 epidemic in China Mainland. medRxiv. 2020.

5. Leung K, Wu JT, Liu D, Leung GM. First-wave COVID-19 transmissibility and severity in China outside Hubei after control measures, and second-wave scenario planning: a modelling impact assessment. Lancet. 2020; 395(10233): 1382-1393.

6. https://www.worldometers.info/coronavirus/

7. Secretaría de Salud. Nuevo coronavirus en el mundo (COVID-19). Comunicado Técnico Diario. 28 de febrero de 2020.

8. Grupo Técnico Interinstitucional del CONAVE. Definiciones operacionales para la vigilancia epidemiológica de COVID-19. Marzo de 2020.

9. Secretaría de Salud. Jornada Nacional de Sana Distancia. Marzo de 2020.

10. Secretaría de Salud. Comunicado del Consejo de Salubridad General. Reconversión hospitalaria COVID-19. 11 de abril de 2020.

11. Secretaría de Salud. Reporte de capacidad total nacional COVID-19. Marzo de 2020.

12. ISSEMyM. Informe anual. Servicios de Salud del Estado de México. 2017.
13. Trilla A. Un mundo, una salud: la epidemia por el nuevo coronavirus COVID-19. Med Clin. 2020; 154(5): 175-177.

14. Wu JT, Leung K, Leung GM. Nowcasting and forecasting the potential domestic and international spread of the 2019-nCoV outbreak originating in Wuhan, China: a modelling study. Lancet. 2020; 395(10225): 689-697.

15. Rothe C, Schunk M, Sothmann P, Bretzel G, Froeschl G, Wallrauch C et al. Transmission of 2019-nCoV Infection from an Asymptomatic Contact in Germany. N Engl J Med. 2020; 382(10): 970-971.

16. Thompson R. Pandemic potential of 2019-nCoV. Lancet Infect Dis. 2020; 20(3): 280.

17. Chen N, Zhou M, Dong X, Qu J, Gong F, Han Y et al. Epidemiological and clinical characteristics of 99 cases of 2019 novel coronavirus pneumonia in Wuhan, China: a descriptive study. Lancet. 2020; 395(10223): 507-513.

18. Lai X, Wang M, Qin C et al. Coronavirus Disease 2019 (COVID-2019) infection among health care workers and implications for prevention measures in a tertiary hospital in Wuhan, China. JAMA Netw Open. 2020; 3(5): e209666. doi: 10.1001/jamanetworkopen.2020.9666.

19. Heneghan C, Oke J, Jefferson T. COVID-19 How many healthcare workers are infected? Centre for evidence-based, University of Oxford; 2020.

20. International Council of Nurses website. High proportion of healthcare workers with COVID-19 in Italy is a stark warning to the world: protecting nurses and their colleagues must be the number one priority. Published March 2020. Accessed March 30, 2020. Available in: https:// www.icn.ch/news/high-proportion-healthcare-workers-covid-19-italystark-warning-world-protecting-nurses-and.

21. Secretaría de Salud de México. Conferencia de prensa. 11 de mayo de 2020.

22. Cap. 10: La administración por objetivos. In: Chabenato I. Introducción a la teoría general de la administración. 8a edición. New York, NY: Ed. McGraw Hill; 2014. p. 171.

23. Hall RH. Organizaciones: estructuras, procesos y resultados. 6a ed. México: Pearson Prentice Hall; 2006.

24. Cap. 13: Teoría de la conducta de la administración. In: Chabenato I. Introducción a la teoría general de la administración. 8a edición. New York, NY: Ed. McGraw Hill; 2014. pp. 257-266.

25. Escudero MJ, Delfín BLA, Arano CRM. El desarrollo organizacional y la resistencia al cambio en las organizaciones. Ciencia administrativa. 2014; 1.

26. Gobierno del Estado de México. Acuerdo para la implementación de medidas para el personal adscrito a las unidades médicas y administrativas del ISSEMyM para enfrentar la situación sanitaria derivada del COVID-19. Marzo de 2020.

27. Youyang Gu. Abaput COVID-19 projections. Massachusetts institute of technology. 2020. Available in: https://covid19-projections. $\mathrm{com} /$ mexico

28. https://www.worldometers.info/coronavirus/

29. Guterres A. Mensaje dirigido por la pandemia COVID-19. Secretario General de la ONU. Marzo de 2020. 